\title{
First reported case of per anal endoscopic myectomy (PAEM): A novel endoscopic technique for resection of lesions with severe fibrosis in the rectum $\square$
}

\section{(이요 $\odot$}

\author{
Authors \\ David Ozzie Rahni ${ }^{1}$, Takashi Toyonaga ${ }^{2,3}$, Yoshiko Ohara ${ }^{4}$, Francesco \\ Lombardo ${ }^{5}$, Shinichi Baba ${ }^{3}$, Hiroshi Takihara ${ }^{3}$, Shinwa Tanaka ${ }^{4}$, \\ Fumiaki Kawara ${ }^{4}$, Takeshi Azuma ${ }^{4}$ \\ Institutions \\ 1 Brown University/Rhode Island Hospital, Rhode Island Hospital, \\ Providence, United States \\ 2 Department of Endoscopy, Kobe University Hospital, Kobe, \\ Japan \\ 3 Department of Endoscopy, Kishiwada Tokushukai Hospital, \\ Kishiwada, Japan \\ 4 Division of Gastroenterology, Department of Internal Medicine, \\ Graduate School of Medicine, Kobe University, Kobe, Japan \\ 5 Emergency Endoscopy Unit, Borgo Trento Hospital, Verona, Italy \\ submitted 28.6.2016 \\ accepted after revision 4.10.2016
}

\author{
Bibliography \\ DOI http://dx.doi.org/10.1055/s-0042-122965 | \\ Endoscopy International Open 2017; 05: E146-E150 \\ (c) Georg Thieme Verlag KG Stuttgart · New York \\ ISSN 2364-3722 \\ Corresponding author \\ Takashi Toyonaga, MD, PhD, Department of Endoscopy, Kobe \\ University Hospital, 7-5-1 Kusunoki-cho, Chuo-ku, Kobe, Japan \\ Fax: +81-78-382-6309 \\ toyonaga@med.kobe-u.ac.jp
}

Background and study aims A 54-year-old man was diagnosed with a rectal tumor extending through the submucosal layer. The patient refused surgery and therefore endoscopic submucosal dissection (ESD) was pursued. The lesion exhibited the muscle retraction sign. After dissecting circumferentially around the fibrotic area by double tunneling method, a myotomy was performed through the internal circular muscle layer, creating a plane of dissection between the internal circular muscle layer and the external longitudinal muscle layer, and a myectomy was completed.

The pathologic specimen verified T1b grade 1 sprouting adenocarcinoma with $4350 \mu \mathrm{m}$ invasion into the submucosa with negative resection margins.

\section{Introduction}

Endoscopic submucosal dissection (ESD) was first performed on superficial gastric lesions and now is widely accepted as a treatment for lesions not only in the stomach, esophagus, and colon but also in the pharynx and even those involving the anal canal [1]. Rectal ESD has no limitation in regards to lesion size [2]. The clinical advantages of rectal ESD include the ability to avoid invasive surgery, stoma and ensure functional prognosis.

In some cases of severe submucosal fibrosis, obtaining en bloc resection can be very difficult. We performed ESD with myectomy, which resulted in accurate pathologic diagnosis of tumor, and hereby introduce a novel endoscopic technique for resection of rectal lesions with severe fibrosis exhibiting the muscle retracting (MR) sign.

\section{Case Report}

A 54-year-old man was found to have a Type 0-Is (Paris Classification) rectal tumor, about $4 \mathrm{~cm}$ in size, visualized $1.5 \mathrm{~cm}$ proximal to the dentate line ( $\boldsymbol{F}$ Fig. 1a). The magnifying NBI classification according to Japan NBI Expert Team (JNET) was Type 2B [3] ( $\triangleright$ Fig. 1b). Crystal violet staining of the lesion showed that it had a Kudo Type Vi high grade pit pattern ( $>$ Fig. 1c). Endoscopic ultrasonography (EUS) revealed tumor extension and fibrosis at the center of the lesion extending through the submucosal layer and ending adjacent to the muscle layer ( $\triangleright$ Fig. 1d). The tumor depth was diagnosed as SM3. The patient vehemently refused surgery and therefore ESD was pursued.

The pocket creation method [4] was used until the center of the lesion was reached and a fibrotic area with probable tumor was found closely adherent to the internal circular muscle layer. That fibrotic area at the center of the lesion exhibited the MR sign or muscle propria underlying the tumor being drawn upward by the tumor to form a triangular shape ( $\mathbf{F i g}$. 2a). This MR sign has been associated with high risk of incomplete removal via ESD [5]. Two submucosal tunnels were then created with a retroflexed view on either side of this fibrotic area to expose the underlying muscle layer and create an adequate dissection plane in the muscle propria ( $\mathbf{F i g . 2 b}$ ). Instead of dissecting between the fibrotic tumor and muscle propria, a myotomy was performed through the internal circular muscle layer, creating a plane of dissection between the internal circular muscle layer and the external longitudinal muscle layer ( $\triangleright$ Fig. 2c). For the myotomy, Flush knife-BT was used with Endo Cut mode of ERBE VIO 300 D. After the 2 rectal wall mus- 

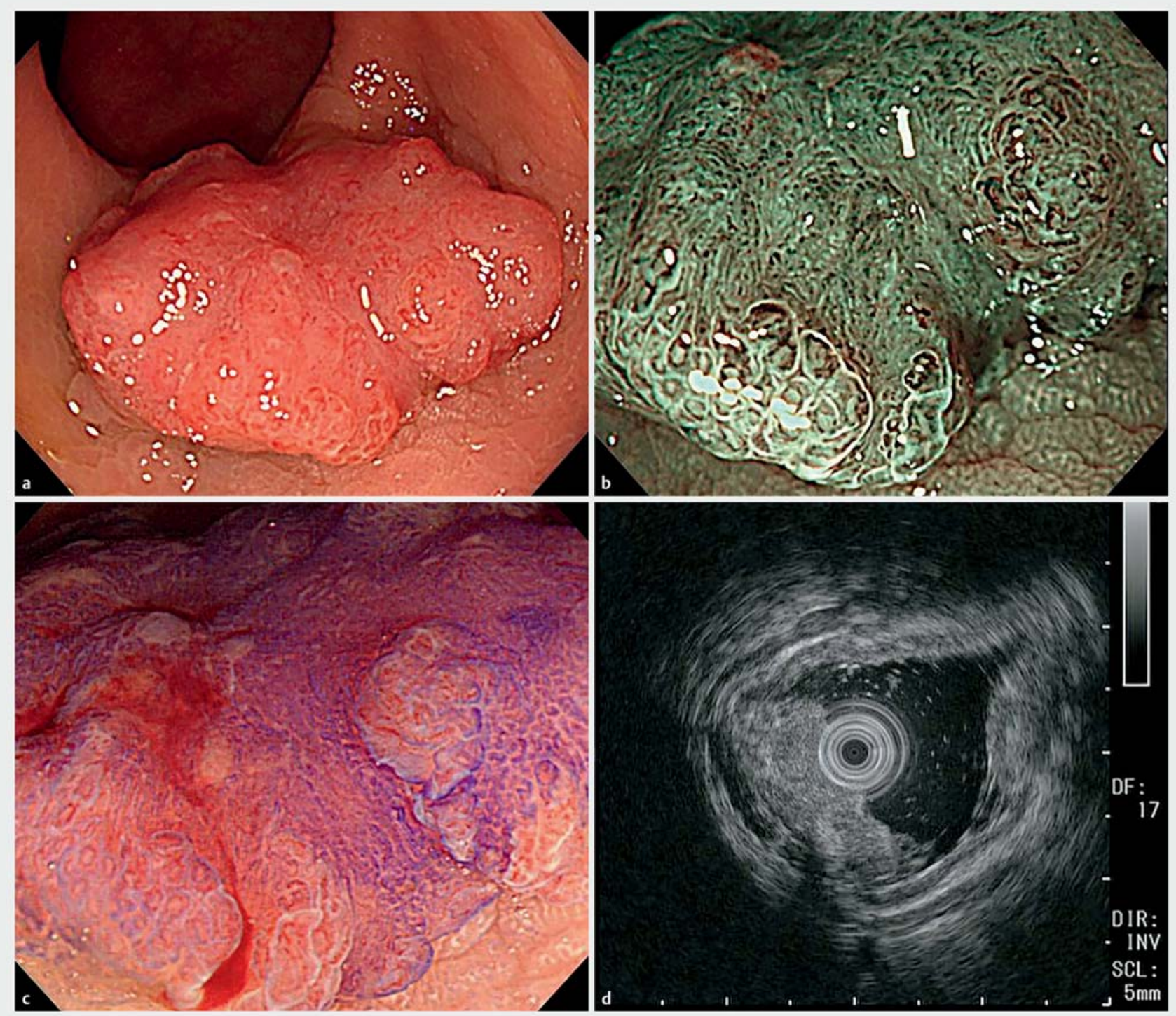

- Fig. 1 a Type 0 -Is rectal lesion, about $4 \mathrm{~cm}$ large. b NBI image showing Type 2B in classification by NBI Expert Team (JNET). c Crystal violet stain showing Kudo Type Vi high grade pit pattern. $\mathbf{d}$ EUS image revealing tumor extension and fibrosis at the center of the lesion extending through the submucosal layer and ending adjacent to the muscle layer.

cle layers were completely separated underneath the fibrotic center, the remaining submucosa on either lateral side was dissected and the tumor was removed along with the underlying internal circular muscle ( $>$ Fig. 2 d, $>$ Fig. 2e, $>$ Fig. 2f) ( $\vee$ Video 1). The pathologic specimen was confirmed to be T1b grade 1 sprouting adenocarcinoma with $4350 \mu \mathrm{m}$ invasion into the submucosa with negative resection margins consistent with a SM3 lesion. There was no lymphatic invasion but there was venous invasion ( $>$ Fig.3). The patient agreed to only adjuvant chemoradiation. Repeat colonoscopy 1 month later revealed almost complete re-epithelization ( $\triangleright$ Fig.4).

\section{Discussion}

ESD is increasingly being recognized in western countries as an effective method of removing a variety of early gastrointestinal tumors throughout the entire gastroenteric tract. ESD provides both a therapeutic and diagnostic modality for management of gastrointestinal tumors. By providing en bloc specimens, precise histopathologic determination of resection margins, tumor extension depth, and potential lymphovascular invasion can help assess curability and determine the next step in management. The largest experience with the technique has been in the upper gastrointestinal tract on intramucosal lesions and those extending to the superficial layers of the submucosa. Even so, ESD is being applied more commonly to colorectal le- 

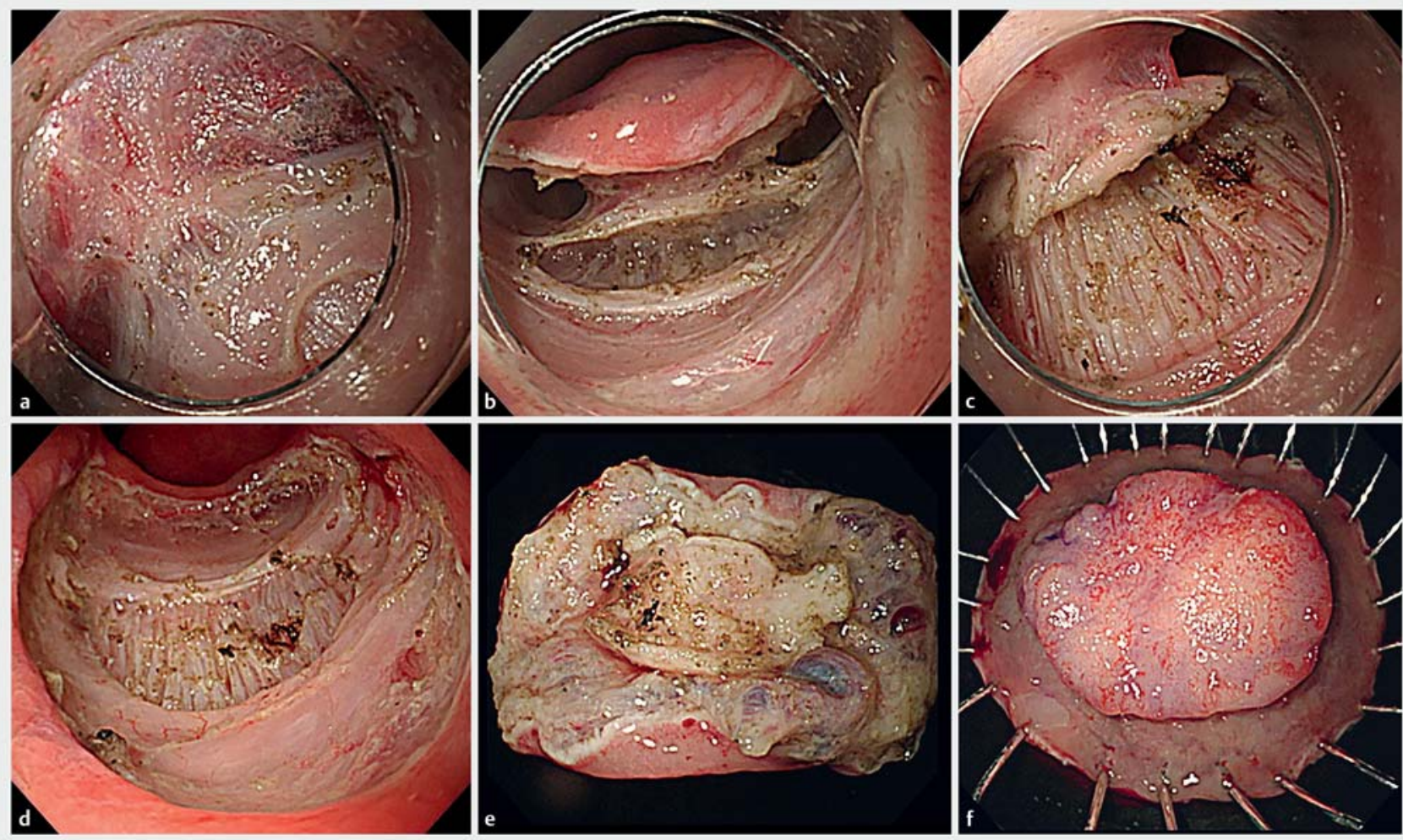

- Fig. 2 a Muscle retraction sign that was found at the center of the lesion. b Two submucosal tunnels on either side of the fibrostic area created to expose the underlying muscle layer. c Dissection between inner circular muscle and outer longitudinal muscle. $\mathbf{d}$ The artificial ulcer left after PAEM. e Macroscopic view of the resected specimen from submucosal side. $\mathbf{f}$ Macroscopic view of the resected specimen from mucosal side.

sions including complex lesions, such as fibrotic, circumferential, and very large ones $(>10 \mathrm{~cm})$.

However, there is no standardized preoperative method of determining the depth of submucosal tumor invasion and it is currently estimated based on a combination of endoscopy, mucosal pattern magnification, endoscopic ultrasonography, and radiologic examination. When the MR sign is present, there are 2 general possibilities, 1 of which is that the tumor has actually gone through deep submucosa into the muscle propria, pre-

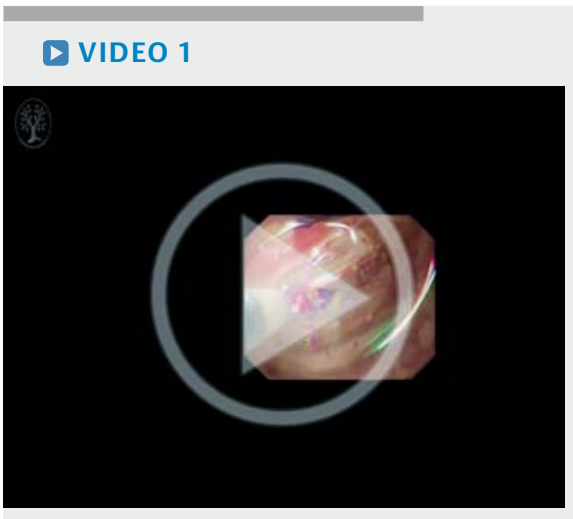

- Video 1: Key video segments of PAEM procedure. cluding ESD [5]. The other possibility, in about one-third of cases, is presence of only fibrosis created by peristalsis. In the latter scenario, ESD is a therapeutic option and patients can avoid unneeded surgery.

One method for achieving $\mathrm{R} 0$ resection in these situations is the pocket creating method (PCM). This method allows definite

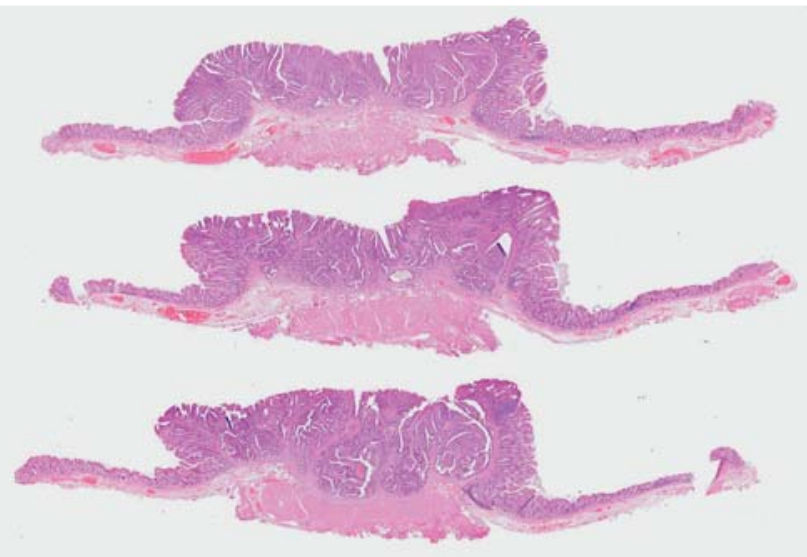

Fig. 3 Pathologic image of lesion that was removed with the internal circular muscle. 


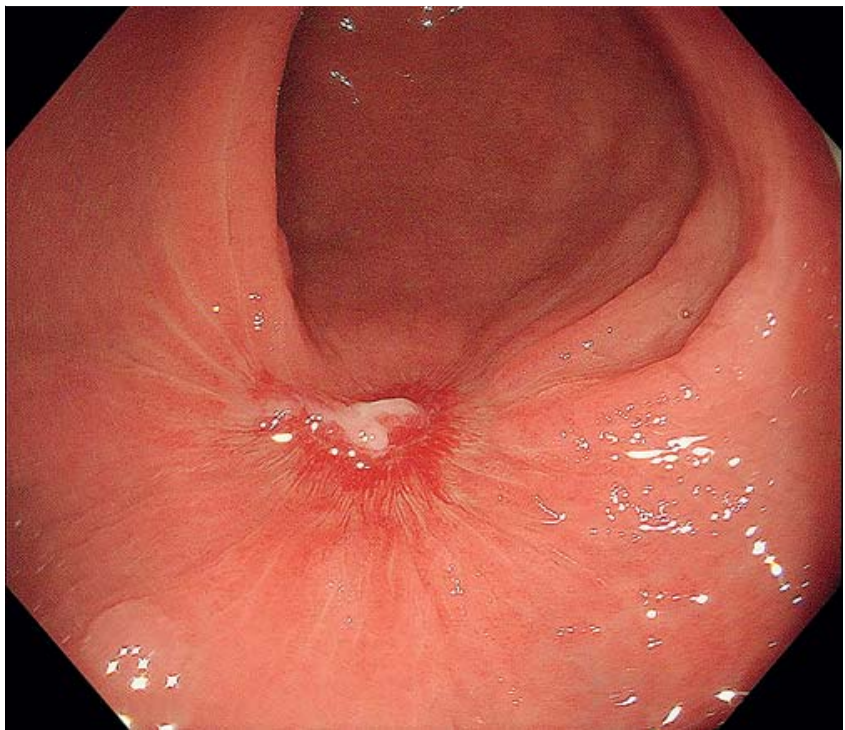

- Fig. 4 Healed rectal site one month after PAEM.

traction in the fibrotic area and dissection precisely just above the muscle fibers using the ESD technique. However, high-level endoscopic skill is needed and, in the case of deep invasion, the risk also exists of positive vertical margin and overlooking lymphovascular invasion.

The other options for these cases are transanal excision (TAE) and transanal endoscopic microsurgery (TEM), which can achieve full-thickness resection. Local excision for appropriate patients is always preferred over sphincter-sparing procedures or abdominal perineal resection, given reduced morbidity, which includes but is not limited to diverting ostomy, genitourinary dysfunction, and loss of anal function [6]. The disadvantage of TAE alone is the high rate of recurrence compared to TEM [7]. TEM is reported to achieve a high $\mathrm{R} 0$ resection rate by full-thickness fashion, and it significantly reduces the need for further abdominal treatment. On the other hand, TEM generally cannot be carried out when the distal tumor margin is within $3 \mathrm{~cm}$ of the anal verge or the tumor is large or protruding, given technical procedural difficulties.

ESD is also utilized in an ever-expanding array of endoscopic procedures as exhibited by peroral endoscopic myotomy (POEM), gastric-POEM, and submucosal endoscopic tumor resection (SET) enabling more experience with muscular propria dissection. Particularly in SET, myectomy is performed in a circular manner at the level between the inner circular and outer longitudinal muscle to avoid damaging the tumor membrane in the submucosa [8].

Difficulties in the cases with positive MR sign mentioned above and experience of endoscopic approach to muscles dissection have led to the idea for and development of PAEM.

In the PAEM procedure, after dissecting circumferentially around the fibrotic area with a double tunneling method, the inner circular muscle is cut in a circular manner, which makes the outer longitudinal muscle clearly visible. The space between the inner circular and outer longitudinal muscles is sparse and suitable traction with the tunneling method makes it easier to dissect this space. As seen in the case presented here, even with SM massive tumor invasion, a definite negative vertical margin can be obtained because the margin includes the inner circular muscle. On the other hand, in POEM and SET, the mucosal layer is left behind as a safety valve [9], but in PAEM, the muscular layer is exposed post-procedure. Delayed perforation into the intraperitoneal cavity, if the indication is limited to the lower rectum, generally does not occur because of the relative thickness of the outer rectal longitudinal muscle. In the current case, no clip closure was carried out and the clinical course was preferable, with the ulcer bed becoming epithelized 6 weeks after PAEM. However, to guarantee further safety, clipping of the defected inner muscle area might be considered. PAEM may be feasible for other organs if a perfect closure can be accomplished. The stomach could be a next candidate for PAEM.

In this article we introduced a novel method of PAEM. The type of cases to which this method should be applied remain to be clarified based on further clinical experience. The best indication may be for cases of a positive MR sign from fibrosis, which requires an easy and complete endoscopic resection. On the other hand, other cases that may lend themselves to PAEM include those with deep submucosal tumor invasion without other high-risk factors such as poorly differentiated type, lymphovascular invasion or budding grade $2 / 3$, which have been proven to have a low risk of lymph node metastasis [10]. In that situation, PAEM will help to accurately diagnose tumor depth. At present, however, there is no modality for distinguishing whether the MR sign is coming from tumor invasion or just caused by mechanical stimulation such as peristalsis. The diagnostic ability of EUS is not so excellent, especially for large protruded lesions. Therefore, it can be considered in cases when the MR sign is positive although surgery must be the first choice for suspected advanced cancer. PAEM should not be performed for MP or deeper lesions, because dissemination would be a risk, if the tumor was harmed during the procedure. It also should not be performed by endoscopists who lack enough experience or cannot distinguish tumor depth or muscle fibers.

Another problem may be a case in which tumor invasion to the outer longitudinal muscle is found after PAEM has started. In that scenario, the space between inner and outer muscle cannot be observed. Such cases would be rare because we do not attempt endoscopic treatment when MP cancer is suspected, but when they are encountered, full-thickness resection with the PAEM technique would be feasible, although further investigation of this is needed; endoscopic treatment also could be suspended in favor of surgery. It also must be noted that a report exist about increased risk of local recurrence in high-risk patients with submucosal rectal cancer than in those with submucosal colon cancer [11]. Therefore, if a patient is confirmed to have high-risk factors other than SM deep invasion, additional therapy such as surgery and chemoradiation should be considered. 


\section{Conclusion}

ESD has been shown to be an effective minimally invasive, lowmorbidity management option for $\mathrm{T} 1$ rectal tumors. In the case presented here, a TI rectal lesion exhibiting a positive muscle retraction sign was treated with ESD via myectomy. The approach included resection of the inner muscular layer, which resulted in an en bloc specimen and, therefore, accurate diagnosis. Further experience is needed with this novel PAEM technique for management of massive submucosa-invading T1 tumors and lesions with severe fibrosis in the rectum in an effort to develop a low-morbidity diagnostic technique and a therapeutic modality option via ESD. We believe this is the first reported case of ESD used per anal myotomy as well as myectomy in removing a rectal malignancy.

Competing interests

Takashi Toyonaga invented the Flush knife-BT in conjunction with FUJIFILM and receives royalties from its sale.

\section{References}

[1] Tamaru Y. Oka S. Tanaka S et al. Endoscopic submucosal dissection for anorectal tumor with hemorrhoids close to the dentate line: a multicenter study of Hiroshima GI Endoscopy Study Group. Surg Endosc 2016; 30: $4425-4431$
[2] Ohara Y. Toyonaga T. Tanaka S et al. Risk of stricture after endoscopic submucosal dissection for large rectal neoplasms. Endoscopy 2016; 48: $62-70$

[3] Sano Y. Tanaka S. Kudo SE et al. Narrow-band imaging (NBI) magnifying endoscopic classification of colorectal tumors proposed by the Japan NBI Expert Team. Dig Endosc 2016; 28: 526 - 533

[4] Hayashi Y. Sunada K. Takahashi H et al. Pocket-creation method of endoscopic submucosal dissection to achieve en bloc resection of giant colorectal subpedunculated neoplastic lesions. Endoscopy 2014; 46: E421 - E422

[5] Toyonaga T. Tanaka S. Man IM et al. Clinical significance of the muscle-retracting sign during colorectal endoscopic submucosal dissection. Endosc Int Open 2015; 3: E246-E251

[6] Banerjee AK. Sexual dysfunction after surgery for rectal cancer. Lancet 1999; 353: $1900-1902$

[7] de Graaf E]. Burger JW. van ljsseldijk AL et al. Transanal endoscopic microsurgery is superior to transanal excision of rectal adenomas. Colorectal Dis 2011; 13: $762-767$

[8] Inoue H. Ikeda H. Hosoya T et al. Submucosal endoscopic tumor resection for subepithelial tumors in the esophagus and cardia. Endoscopy 2012; 44: $225-230$

[9] Sumiyama K. Gostout C]. Rajan E et al. Submucosal endoscopy with mucosal flap safety valve. Gastrointest Endosc 2007; 65: 688- 694

[10] Yoshii S. Nojima M. Nosho K et al. Factors associated with risk for colorectal cancer recurrence after endoscopic resection of T1 tumors. Clin Gastroenterol Hepatol 2014; 12: 292 - 302.e293

[11] Ikematsu H. Yoda Y. Matsuda T et al. Long-term outcomes after resection for submucosal invasive colorectal cancers. Gastroenterology 2013; 144: 551 - 559 ; quiz e514 